\title{
Ethics and End of Life Care: the Liverpool Care Pathway and the Neuberger Review
}

\begin{abstract}
The Liverpool Care Pathway for the Dying has recently been the topic of substantial media interest and also been subject to the independent Neuberger Review. This review has identified clear failings in some areas of care and recommended the Liverpool Care Pathway be phased out. I argue that while the evidence gathered of poor incidences of practice by the Review is of genuine concern for end of life care, the inferences drawn from this evidence are inconsistent with the causes for the concern. Seeking to end an approach that is widely seen as best practice and which can genuinely deliver high quality care because of negative impressions that have been formed from failing to implement it properly is not a good basis for radically overhauling our approach to end of life care. I conclude that improvements in training, communication, and ethical decision-making, without the added demand to end the Liverpool Care Pathway would have resulted in a genuine advance in end of life care.
\end{abstract}




\section{Ethics and End of Life Care: the Liverpool Care Pathway and the Neuberger Review}

\section{Introduction}

The Liverpool Care Pathway for the Dying (LCP) has recently been the topic of substantial media interest and subject to the independent Neuberger Review (NR).[1] This Review identified areas where there are clear failings of care requiring attention but also raised questions about the very nature of end of life care. The somewhat unexpected recommendation of NR is that the use of the LCP be phased out and replaced with individualised patient care plans. This recommendation is particularly surprising given the widespread, recent endorsements from professional bodies the LCP has received,[2-4] as well as it being the subject of numerous studies which have highlighted the improvements in end of life care that come from using the LCP.[5-8]

Although the evidence gathered surrounding incidences of poor practice by NR is of genuine concern, the inferences drawn from this evidence seem out of step with the causes of these concerns. These concerns are largely based either on misconceptions about, or improper implementation of, the LCP. Part of this lack of proper implementation includes the failure to make good ethical decisions about patient care. The recommendation from NR that we should prioritise improving the quality of end of life care is certainly to be welcomed. However, if the ultimate aim in end of life care is to actually provide, as well as reassure patients that they will receive, good quality care in the last days or hours of their life then enhancing training and understanding, together with continued research into end of life care, would be a better solution than abandoning the LCP approach.

\section{What is the LCP designed to do?}

Given many of the concerns about end of life care outlined in NR have arisen from misconceptions about what the LCP is designed to do, it is important to first clarify its nature and aims. The genesis of the LCP comes from a desire to transfer the best practice for care of the dying from the hospice to the hospital setting.[9] This move is 
of the utmost importance for improving general end of life care, as most deaths occur in hospital settings and relatively few deaths occur in hospices.[10] There is widespread agreement that before the LCP, poor care and suffering was the norm for patients dying in hospitals, with ad hoc guidance and support for clinical teams.[11]

The role of the LCP is to highlight areas of importance and to provide advice in general terms as to approaches to delivery of care and the expected outcome of care delivery.[12] As a framework, the LCP is only meant to support the professional in this area and is not intended as a substitute for clinical judgement or for ethical decision-making. Instead, it is meant to allow care to be tailored to individual patient needs - something called for in NR with individual patient plans - and includes a wide range of aspects of care, such as the physical, psychological, social and spiritual requirements of a dying person. These goals of care are expressed as a series of desired outcomes for patients and their relatives or carers, not a series of processes which must be applied. What it does not provide is a rigid set of guidelines that have to be followed in each individual case. The use of the LCP in clinical practice therefore has to be supplemented with an awareness of ethical decisionmaking and good communication of these decisions.[13: 62] Treating the LCP as if it gave a single 'one size fits all' guide to end of life care, failing to engage in good ethical decision-making, and failure to communicate the combined clinical and ethical reasoning effectively to colleagues, patients and their relatives would constitute a failure to implement the LCP as it was intended. The mistaken view that the LCP provides some sort of generic protocol which provides a specific set of instructions for end of life care that exactly meets the needs of every individual patient is at the heart of the concerns raised by NR.[1: 47]

\section{Concerns over implementation of the LCP}

Perhaps the most dominant concern throughout NR is that hospital staff are implementing the LCP poorly, leading to cases of inadequate end of life care in the hospital setting. Some concern cases where there is a lack of training or a basic misunderstanding of what the LCP is meant to do. Other cases seem to be of a more fundamental failure to adhere to what would be basic ethical decision-making (i.e. 
utilising common-sense morality to identify ethical problems and applying fundamental principles, such as respect, care and compassion, to determine actions). One might expect that given such cause for concern, an entirely understandable outcome would have been for the NR to recommend better training in the use of the LCP; something that the GMC and BMA have already called for.[14, 15] An additional welcome recommendation would have been for better training in ethical decision-making for staff involved in end of life care, given the heavy focus in NR on cases where there appears to be a lack of good communication, compassion, or treating patients with dignity. What seems to be far too extreme, however, is the recommendation by NR that on these grounds the LCP should be phased out.

One reason why this seems too extreme is that end of life care in the UK is of a quality that is world-leading, recently being ranked as having the best overall palliative care in the world.[16] Moreover, one of NR's conclusions was that:

"[I]n the right hands, the Liverpool Care Pathway can provide a model of good practice for the last days or hours of life for many patients...But it is clear that, in the wrong hands, the LCP has been used as an excuse for poor quality care." [1: 47]

However, to recommend from this position that the LCP be phased out is to make a seemingly invalid inference. By analogy, one might construct a similar argument for many different treatments by claiming that, for example, the use of morphine should be phased out as a painkiller in medicine because its correct use is beneficial but some people incorrectly use it, or that insulin should no longer be used as a treatment for diabetes because of its harmful incorrect use. At very best, such an inference may seem to be of the type one adopts as a result of precautionary measures that result from concerns over the possibility of extreme negative consequences and high likelihood of misuse (such as arguments that restrict the use of morphine outside of a controlled medical environment). It therefore seems a suspect rationale has been offered in calling for the end to the LCP, where we stand to lose a high quality approach to care on the grounds that it can be misapplied by those not properly trained in its use. 
The recommendation seems all the more peculiar because NR indicates that "the LCP entirely reflects ethical principles that should provide the basis of good quality care".[1: 8] What presents itself in NR as the unifying concern underpinning the recommendation to phase out the LCP is that poor application and communication with patients, families and carers when it comes to implementing the LCP has led to the negative impressions surrounding it. These negative impressions have been greatly enhanced by media coverage, with reports from palliative care doctors that "[n]egative press regarding the LCP has caused additional distress for relatives at an already distressing time when their loved one is dying."[17]

In order to attempt to make sense of the NR recommendation, closer examination is needed of whether there are specific ethical problems with the LCP stemming directly from its implementation which might justify its cessation, rather than some form of failure to adequately train staff in end of life care generally. NR identifies a number of key concerns with the LCP, including: decision-making, consent, involvement in the care plan, hydration and nutrition, sedation and painmanagement, and the use of financial incentives.[1: 6-8] Looking across a range of these issues it is apparent that there is little, if any, basis for seeking an end to the LCP that can be derived from concerns specifically attributable to it.

\section{Problems with decision-making and communication}

The first major concern over "sloppy and unmonitored decision-making" [1:21] is not attributable to the LCP itself, which advises about the importance of the decisionmaking process, but is rather a manifestation of poor basic practice. It is even acknowledged that:

"[NR] fully recognises the valuable contribution that approaches like the LCP have made in improving the timeliness and quality of clinical decisions in the care of dying patients."[1:21].

The same is true of an associated concern surrounding consent, where there is "misunderstanding and uncertainty" [1:23] over whether deciding to implement the LCP is a treatment decision that requires the patient's consent (or in their best interests where the person lacks capacity). Although the LCP does not in any way impede or prevent the proper understanding and application of consent there is a 
more fundamental error in place because the LCP is not a treatment in itself but a guide to treating. A patient is not placed on the LCP as a treatment option. Rather, the LCP is utilised to help medical teams make better decision choices once a patient's prognosis is that they have only a few days or hours of life left. Treatment options that might be offered as appropriate within the LCP framework would still require consent from a patient, their proxy or to be in their best interests, accordingly. Although the phrase "being placed on a care pathway" might give the impression that a patient is being placed on a predetermined course of treatment, it is instead similar to health care practitioners following guidelines in any specialist area of medicine. We would not think for a patient with, for example, a bone fracture that their consent was needed to agree to their treating practitioner to follow professional orthopaedic consultancy guidelines in forming views about various appropriate treatment options.

Much of the recent controversy found in the media surrounded how families felt in the face of poor communication surrounding the use of the LCP.[18] Not involving carers in discussions surrounding its implementation or simply not even discussing that a relative was dying when their care was supported by the LCP are all cases where communication between professionals and patients' families and carers was undoubtedly poor. It is an important question as to how much information family members or carers should be privy to when considering the treatment of any individual patient. Without the explicit agreement of the patient, releasing information about treatment decisions to others might be considered a breach of confidentiality and a failure to respect their autonomy. The simple fact that a patient is believed to be in the last hours or days of life is not, in itself, sufficient reason to ignore these principles. However, there are other factors in the end of life setting which might also need to be taken into account. The most significant of these would be if the patient were no longer sufficiently competent. Here, consulting family members or carers would be an important means of determining what approach to treatment would be in the best interests of the patient. It may also be prudent if the treatment is likely to render the patient unconscious or incapacitate them in some other way, so that the family can prepare to be consulted about further treatment options. In these cases, there is a clear requirement for good communication in order to meet basic duties. 
It is, however, important to recognise that a careful line must be trodden in cases where a patient who is still autonomous is being treated. Although relatives and carers of the patient might have genuine, altruistic reasons to be concerned about their care and treatment, what should and should not be discussed with them is still a matter of good ethical judgement. Whether there is a case to treat end of life care differently from other forms of medical care has not been established in NR, hence discussion of a patient's treatment is far from a right that families and carers have. Nevertheless, prudent communication as to the nature of end of life care would help prevent misconceptions about treatment that could develop into scandalised comments.

\section{Concerns over hydration and nutrition}

Perhaps some of the most startling - and frequent - accusations levelled against the use of the LCP have surrounded hydration and nutrition of patients.[1:26] Hydration has proved to be an important issue, partly because of confusion over LCP guidance that it may be acceptable to not hydrate patients with intravenous or subcutaneous fluids, and partly because of reports that patients were refused liquids even though they specifically requested them, were deemed to be desperate for them by relatives or carers, or that the refusal of a particular drink at a particular time led to the inference by hospital staff that a patient no longer wanted to receive fluids at all.[1: 26-7] There are a number of separable concerns here. The first is a general concern as to whether it is ever acceptable to not provide artificial hydration for patients who can no longer take fluids by mouth. This situation can lead to the belief that such refusal not only intentionally hastens the death of the patient [1:27] but also that it leads to an agonising death with the unrelieved suffering of thirst:

\footnotetext{
"The urge to drink when thirsty is very powerful and basic. Good mouth care if the patient simply has a dry mouth may well be sufficient, but to deny a drink to a thirsty patient is distressing and inhumane."[1:28]
}

It would therefore seem that to deny fluids was a serious breach of a duty to care for a patient and difficult to see how this could be in the best interests of any patient. 
However, a blanket policy of always providing artificial hydration is as ethically indefensible as a blanket policy not to provide such hydration. The key is to distinguish between cases where a patient is dying from an underlying disease from cases where they are dying because they are unable to adequately maintain their hydration. Some patients can benefit substantially from artificial rehydration but there are cases where the harms outweigh the potential benefits. Accordingly, the appropriateness of hydration, clearly established within LCP guidance, "should be judged on a day-to-day basis, weighing up the potential harms and benefits."[13: 69] There are good reasons why artificial hydration might not be given or even why it might be withdrawn if it was being provided. This might be due to the welldocumented lessening of desire for food and drink in the end stages of life or the thirst being caused by medication rather than lack of hydration, where artificial hydration would be only a burdensome and unnecessary intrusion. Other cases where the patient is suffering due to an excess of fluids, such as excess respiratory secretions, oedema, etc. also provide a strong clinical indication that hydration should not be given. The point is that where hydration is not wanted by the patient and not physiologically beneficial to the patient, other methods of controlling symptoms of thirst are likely to be less harmful.

This is not to say that all patients in the last hours or days of life would not benefit or find comfort in taking fluids. There is nothing in the LCP that requires a cessation of fluids where there is no clinical requirement not to provide them and there is no doubt that there is a basic, instinctive expectation on the part of families that hydration and nutrition would be provided as part of basic patient care. Issues such as family misunderstandings over the appropriate use of hydration can be accounted for through a failure to properly communicate what might appear as a counterintuitive aspect to end of life care. This is widely recognised in medical literature and the NR acknowledged that a systematic review of the literature and studies evaluating clinically assisted hydration showed "no clear benefit to either length or quality of life".[1: 26]

More emphasis, however, was placed on the number of cases where hydration was not provided to patients due to an incorrect understanding of hydration in end of life care.[1: 27] The discovery that staff were refusing patients oral drinks when 
desperate for them was rightly deemed appalling by the NR, although it is notable that, once again, it is not the LCP that is deemed to be the root cause of the problem. If anything, this recognises the exact opposite - that it is by not following the LCP that patients receive poor end of life treatment. Perhaps the only criticism that can genuinely be levelled is the NR panel's consideration that the language of the current LCP does "not go far enough" [1:26] to advise that the default course of action be that patients should be supported with oral hydration and nutrition unless there is strong reason not to do so. However, this surely means that what is required is better training for hospital staff as to how to properly interpret the LCP rather than to view the cause of the problem as the LCP itself.

\section{Concerns over deliberate hastening of death}

The gravest source of concern identified in NR is the belief that the LCP can be used to deliberately hasten the death of patients. There are two notable ways this might potentially happen: through the withdrawal or withholding of hydration and nutrition or through the use of pain relieving medication, such as morphine. Sometimes, the hydration and medication are connected, in that strong sedation (such as continuous deep sedation) can subsequently allow death by dehydration if no artificial hydration is put in place.

It is the general aim of the LCP that patients are allowed to die in as peaceful a state as possible, with two of the specific goals identified in their ongoing care assessment as: (a) - the patient does not have pain, and (b) - the patient is not agitated.[19] In these cases, the use of morphine to control pain or the use of sedatives to calm agitated or distressed patients would be well within the bounds of clinical acceptability. However, two concerns arising from this are identified in NR. One is the feeling of some patients' relatives that the use of diamorphine had directly killed the patient and the other was that syringe drivers containing morphine were put in place through simply believing this was the 'next step' required by the LCP.[1: 29-30]

The first of these is a misconception: relieving pain using medication is not a form of euthanasia. For this to be euthanasia there would have to be the intention to kill or hasten the death of the patient but the intention in these cases is to improve the comfort of the patient.[20] Although it is recognised even in LCP guidelines that it is 
difficult to know for sure whether it was the prescribed injection or the underlying disease that caused the death of the patient, without the clear intention to bring about their death it cannot be euthanasia.[13: 71] Both the NR and the LCP also acknowledge that the appropriate use of opioids in symptom control has no affect on lifespan and any continuous use of them or sudden increase in dosage should be regularly reviewed.[13: 71]

The second concern about the use of syringe drivers raises a number of issues. The first is that relatives and carers giving evidence to NR recounted how they left the patient in a peaceful state only to return to find them with a syringe driver fitted and unable to communicate. This included one case where a family was under the impression that the patient had been overmedicated in order to allow him to die from dehydration and others where concern was raised over the cessation of a patient's regular medicine for an established condition, such as insulin for their diabetes.[1: 29-30] Whilst I fully accept the view in NR that much of the distress caused under these circumstances would have been "mitigated by better communication" [1: 30] so that relatives and carers would be less likely to link the changes in medication or the cessation of unnecessary or burdensome treatments with the cause of patient death, this is still a matter founded on third party confusion rather than innate problems with the LCP. LCP guidelines are clear that good communication with relatives and carers, where appropriate, is called for. It is not clear, however, that these problems over communication wouldn't have arisen regardless of whether a care pathway was being used to support care or not. Poor communication is not a desirable feature of any medical care. That hospital staff were purportedly using a care pathway that explicitly states the importance of good communication highlights an underlying problem over care provision in hospitals rather than with the LCP, as highlighted in three rounds of the National Care of the Dying Audit-Hospitals.[21]

Furthermore, the NR raised concerns that those deciding on the drugs to be given to patients had "not received training in their use to an acceptable level of competence" and that patients were being placed on syringe drivers containing morphine when morphine was not the right drug.[1: 30] The use of syringe drivers in this way is not justifiable given that the treatment is, by definition, unnecessary. Accordingly, NR recommends that before their use, discussion should take place with the patients, 
their relatives or carer, and the reasoning documented.[1: 30] This recommendation is, however, the advice already laid out in the LCP, where, for example, it is stated that: "It is vital that the patient and their family or carers are involved in the decision to start a syringe driver."[22: 51].

\section{Wider ethical issues}

My central response to the concerns so far addressed is that NR is not recommending anything that is not already outlined as good practice in LCP materials. There remain, however, a range of ethical issues in NR surrounding the wider status of the LCP. Of these, the most pressing is the problem of research underpinning the LCP. Although the LCP is an evidence-based framework founded on high quality medical practice in palliative care, it is noted in NR that there is a lack of strong evidence base comparing pathways for dying with other forms of care and how factors such as the setting (acute hospitals rather than hospices) can result in better or worse implementation.[1: 17]

The recommendation in NR that additional funding be devoted to end of life care is to be warmly welcomed; as there is no doubt that increased research would benefit and inform practice in end of life care. Further research would also have the benefit of underpinning confidence in the approach being taken in end of life care amongst professionals and the wider public. Nevertheless, as lack of research into end of life care is a global problem, there seems little sense in phasing out the most rigorously explored framework and replacing it with another approach that itself has not been subject to research. Furthermore, there are innumerable challenges to overcome in conducting research in the form of robust randomised controlled trials in palliative care which has led to recent research focusing on developing new and innovative research methodologies that are suited to the complications of assessing dying and unconscious patients.[23] The need for research is certainly paramount but it is also consistent with the continued use of the LCP which can continue to be adapted and improved as research results are established.

A second issue is that of financial incentives being provided for each patient placed on the LCP. This was implemented with the intention of incentivising institutions to undertake perceived best practice in their approach to end of life care but the view of 
NR was that it led to a rise in fears about hastening death and encouraged a 'boxticking' approach rather than one of clinical judgement.[1: 34] Although neither are innate problems with the LCP, they are sufficient to arouse suspicion where confidence has already declined or to encourage poor implementation of a system designed to focus on excellence of care rather than the financial vicissitudes of an institution. Therefore the NR recommendation that some alternative means of funding palliative care, ideally as a core service in order to relieve the cost pressures on an institution in this area, would be an entirely welcome addition to supporting the proper implementation of the LCP.

The final salient issue is the concern over the perceived promotion of the LCP as a form of 'brand', whose very name has led to confusion.[1: 16] The term 'care pathway' has led to misconceptions as to the status of the LCP, with is being seen as a protocol rather than an approach to care. This misconception also extends to relatives of patients, who have perceived 'pathway' as meaning something like a 'route to death'. Although some of this confusion was seen to be shared with $\mathrm{DoH}$ and NICE descriptions of what constitutes a 'pathway', there remains a genuine underlying problem as to how approaches to the end of life care are presented. When the reputation of a widely referred to approach such as the LCP is (rightly or wrongly) tarnished, there will inevitably be a knock-on effect by association that diminishes confidence and adds to the difficulties in understanding end of life care amongst professionals, patients and relatives alike. The recommended change in terminology from LCP to "end of life care plan" would be a simple but important means of preventing these misconceptions.[1: 47]

Although a change in terminology might have the effect of reducing branding concerns, the more substantive aspect to the NR recommendation that the LCP be replaced with this "end of life care plan" should be treated cautiously. Despite such individualised plans being entirely consistent with LCP guidance,[24] if the only alteration is that of a name (from "pathway" to "plan") this "plan" has the potential to still be mistakenly seen as "equally prescriptive and task like".[25] This would bring with it the danger of burying a crucial element that NR has brought to light as a vitally needed change: that guidance in this area should not be seen as a prescriptive set of rules to follow dogmatically. 


\section{What do we want from end of life care?}

Although it is unfortunate that the scandal which has arisen through public and professional misconceptions surrounding the LCP has tainted its name and diminished confidence in its use, the issues highlighted in NR act as a warning that end of life care is seen as publically important and that good practice surrounding end of life care is vital for its success. The wider discussion of end of life issues NR invites are essential to overcome a perceived societal taboo concerning death and dying.[26] Whilst the conclusion drawn by NR seems questionable, it has challenged us to consider what might constitute the best possible care for the dying. This is an interesting question but it is unlikely to be achieved without the developments and guidance that the LCP has provided in transferring excellent hospice care to the general setting. The experiences of relatives and carers highlighted in NR have also indicated that something the public wants is a level of reassurance that people are allowed to die in an environment where they receive good quality care in a way that is understandable and delivered by caring, compassionate staff. The route to this seems to be better communication, even where clinical care is delivered appropriately. The longer term goal, alongside this, is to advance our research into palliative care to give both professionals and the public a firmer foundation and confidence in whichever approach they employ.

Palliative care is certainly wider than the LCP itself. The NR has taken us both one step closer and one step further away from the goal of achieving the highest quality end of life care. It has taken us closer by helping to break a long-standing taboo that end of life issues are not widely and openly discussed, by highlighting reasons why there are failings in providing high quality care that are very entrenched and go beyond the failure to properly implement a particular approach, and by calling for more emphasis to be placed on this area in terms of training, support and development. It has also taken us a step further away by calling for the phasing out of a care pathway that is widely held to be an example of the very best approach to palliative care. Were NR to have made the recommendations about improvements in training, communication, and ethical decision-making without the added demand to end the LCP, a considerable advance in end of life care could have been the result. 


\section{REFERENCES}

[1] Neuberger J. More Care Less Pathway: A Review of the Liverpool Care Pathway. Crown Copyright; 2013

https://www.gov.uk/government/uploads/system/uploads/attachment data/file/21245 0/Liverpool Care Pathway.pdf (accessed July 2013)

[2] Price, C. ARM calls for Liverpool Care Pathway to be included in medical school curriculums. Pulse. 26/06/13.

http://www.pulsetoday.co.uk/clinical/therapy-areas/elderly-care/arm-calls-for-

liverpool-care-pathway-to-be-included-in-medical-school-

curriculums/20003425.article\#.UebLhhNwYdU (accessed July 2013)

[3] NHS. The route to success in end of life care - achieving quality in acute hospitals. National End of Life Care Programme. 2010.

http://www.endoflifecare.nhs.uk/search-resources/resources-

search/publications/imported-publications/the-route-to-success-in-end-of-life-care-

achieving-quality-in-acute-hospitals.aspx (accessed July 2013)

[4] General Medical Council. Treatment and care towards the end of life: good practice in decision-making. 2010.

http://www.gmc-uk.org/guidance/ethical guidance/end of life care.asp (accessed July 2013)

[5] Veerbeek L, Zuylen LV, Swart SJ, et al. The effect of the Liverpool Care Pathway for the dying: a multi-centre study. Palliat Med 2008; 22:145-151.

[6] Costantini M, Pellegrini F, Di Leo S, et. al. The Liverpool Care Pathway for cancer patients dying in hospital medical wards: A before-after cluster phase II trial of outcomes reported by family members. Palliat Med 2013. Published Online First 7 May 2013. doi: 10.1177/0269216313487569

[7] Clark JB, Sheward K, Marshall B, et al. Staff perceptions of end-of-life care following implementation of the Liverpool Care Pathway for the Dying Patient in the acute care setting: a New Zealand perspective. J Palliat Med 2012; 15 (4):468-73

[8] Mayland CR, Williams EMI, Addington-Hall J, Cox TF, Ellershaw JE.Does the 'Liverpool Care Pathway' facilitate an improvement in quality of care for dying cancer patients? Br J Cancer 2013; 108:1942-1948.

[9] Marie Curie Palliative Care Institute Liverpool. Statement Regarding LCP Review Publications. 2013. http://www.liv.ac.uk/media/livacuk/mcpcil/documents/Statement,,LCP, review, 15, July,2013.pdf (accessed July 2013). 
[10] Office for National Statistics. Deaths Registered in England and Wales. 2012. http://www.ons.gov.uk/ons/rel/vsob1/death-reg-sum-tables/2012/sb-deaths-firstrelease--2012.html\#tab-Deaths-Registered-in-England-and-Wales--2012 (accessed July 2013).

[11] Mills M, Davies HTO, Macrae WA. Care of dying patients in hospital. BMJ 1994; 309:583

[12] Overill S. The development, role, and integration of integrated care pathways in modern day healthcare. In Ellershaw J, Wilkinson, S, eds. Care of the Dying: A Pathway to Excellence. Oxford: Oxford University Press. 2003: 2-10.

[13] Thorns A, Garrard E. Ethical issues in care of the dying. In Ellershaw J, Wilkinson, S, eds. Care of the Dying: A Pathway to Excellence. Oxford: Oxford University Press. 2003: 62-73.

[14] BMA. Call to improve care pathway training. News Views and Analysis. 18 May 2013.

http://bma.org.uk/news-views-analysis/news/2013/may/call-to-improve-carepathway-training (accessed July 2013)

[15] General Medical Council. Treatment and care towards the end of life: good practice in decision-making. 2010.

http://www.gmc-uk.org/guidance/ethical guidance/end of life care.asp (accessed July 2013)

[16] The Economist. Quality of Death: A Ranking of Care for the Dying By Country. The Economist. 14/07/2010.

http://www.economist.com/node/16585127.

[17] Chinthapalli K. The Liverpool care pathway: what do specialists think? BMJ 2013,346:f1184

[18] Phillips, M. Care? No, this is a pathway to killing people that doctors deem worthless. Daily Mail Oct 142012.

www.dailymail.co.uk/debate/article-2217748/Care-No-pathway-killing-peopledoctors-deem-worthless.html (accessed July 2013)

[19] LCP Model Pathway -UK - Core documentation. October 2012: 19. http://www.sii-mcpcil.org.uk/media/10843/lcp\%20core\%20documentation.pdf

[20] Garrard E, Wilkinson S. Passive Euthanasia. J Med Ethics 2005; 31: 64-68.

[21] Marie Curie Palliative Care Institute Liverpool. National Care of the Dying Audit - Hospitals (NCDAH) Generic Reports (Rounds 1, 2 and 3). 2007; 2009; 2012. http://www.sii-mcpcil.org.uk/lcp.aspx?s=3\#lcpContentAnchor (accessed July 2013) 
[22] Glare P, Dickman A, Goodman M. Symptom control in care of the dying. In Ellershaw J, Wilkinson, S, eds. Care of the Dying: A Pathway to Excellence. Oxford: Oxford University Press. 2003:42-61.

[23] OPCARE9. OPCARE9: A European Collaborative to optimise research for the care of cancer patients in the last days of life. 2011.

http://www.mcpcil.org.uk/media/Doc\%204\%200PCARE9\%20Report.pdf (accessed Dec 2013)

[24] Marie Curie Palliative Care Institute Liverpool. Advanced Chronic Kidney Disease - care for the dying patient: A guide for patients and carers and A guide for health care professionals. 2010.

http://www.sii-mcpcil.org.uk/publications.aspx

[25] Borgstrom E. What's in a name? From pathways to plans. BMJ 2013;347: f4568.

[26] BMA. Dying with dignity. News Views and Analysis. 21 January 2013. http://bma.org.uk/news-views-analysis/news/2013/january/dying-with-dignity (accessed July 2013) 